Progress Report

DOE/ER/45651

\title{
Local Atomic Structure of Semiconductor Alloys Using Pair Distribution Function Analysis
}

Report for the grant period September 1, 2001 through August 31, 2002

by S. J. L. Billinge and M. F. Thorpe

Physics \& Astronomy Department and

Center for Fundamental Materials Research

Michigan State University

East Lansing, MI 48824-2320

USA Project manager - Dr. Robert J. Gottschall

\section{Prepared for THE U.S. DEPARTMENT OF ENERGY AGREEMENT NUMBER} DE-FG02-97ER45651

This report was prepared as an account of work sponsored by the United States Government. Neither the United States nor the Department of Energy, nor any of their employees, nor any of their contractors, subcontractors, or their employees, makes any warranty, express or implied, or assumes any legal responsibility for the accuracy, completeness, or usefulness of any information, apparatus, product or process disclosed or represents that its use would not infringe privately-owned rights. 


\section{Abstract}

We have been taking advantage of recent experimental developments, which involve utilizing diffraction data from $\mathrm{x}$-rays or neutrons out to very large wave-vectors, to obtain a detailed structural characterization of semiconductor alloys. This approach allows an accurate Pair Distribution Function (PDF) to be obtained to $20 \AA$ and beyond and reveals the local structure of the alloy directly. These data can be modeled explicitly to learn about local correlations and short-range order in materials. We are combining theory, modeling and experiments to study a range of materials from semiconductors to thermoelectrics and proton conductors. 


\section{Progress Report}

This is a progress report for the period $1^{\text {st }}$ September 2001 to $31^{\text {st }}$ August 2002.

\section{Personnel supported on the grant}

Professor Simon Billinge, one month summer salary

Professor Michael F. Thorpe, one month summer salary

Dr. Valeri Petkov, post doctoral associate, $50 \%$

Dr. Marek Schmidt, post doctoral associate, $100 \%$

Ahmad Masadeh, graduate student, 50\% summer 2002

Hassan Yavas, graduate student, 100\% summer 2001 and 2002

\section{Products published or submitted since 2000}

\section{Conferences/symposia organized}

1. Impact of Scattering on Nanoscience and Nanotechnology, Symposium at the 2001 American Crystallographic Association Annual Meeting, Los Angeles, CA.

2. From Semiconductors to Proteins: Beyond the Average Structure Traverse City, MI, July 28-August 1, 2001.

3. Real-Space Pair Distribution Function Methods PDF workshop at the 2000 American Crystallographic Association Annual Meeting, Los Angeles, CA.

4. Protein Flexibility and Folding Workshop held in Traverse City, MI, August 2000.

5. Phase Transitions and Self-Organization in Electronic and Molecular Materials, Workshop held ion Cambridge, July 2000.

6. Properties and Applications of Amorphous Materials NATO-ASI held in Sec, Czech, 2001.

\section{Book}

1. S. J. L. Billinge and M. F. Thorpe, Editors, From Semiconductors to Proteins, Kluwer, New York, (2002).

2. L. A. Kuhn and M. F. Thorpe Protein Flexibility and Folding (Elsevier Science Inc., 2002).

3. M. F. Thorpe and J. C. Phillips) Phase Transitions and Self-Organization in Electronic and Molecular Materials (Kluwer Academic/Plenum Publishers, New York, (2000).

4. M. F. Thorpe and L. Tichy Properties and Applications of Amorphous Materials NATO-ASI series (Kluwer Academic Publishers, (2001).

Articles

1. V. Petkov, S. J. L. Billinge, T. Vogt, A. S. Ichimura and J. L. Dye, Structure of intercalated Cs in zeolite ITQ-4: an array of metal ions and electrons confined in a pseudo-1D nanoporous host, Phys. Rev. Lett., to be published (2002).

2. Th. Proffen, V. Petkov, S. J. L. Billinge and T. Vogt, Chemical short range order obtained from the atomic pair distribution function, Z. Kristallogr. 217, 47 (2002).

3. V. Petkov and S. J. L. Billinge, From crystals to nanocrystals: semiconductors and beyond, In From semiconductors to proteins: beyond the average structure, (Kluwer/Plenum, New York, 2002), S. J. L. Billinge and M. F. Thorpe, Eds., pp. 153. 
4. M. F. Thorpe, V. A. Levashov, M. Lei and S. J. L. Billinge, Notes on the analysis of data for pair distribution functions, In From semiconductors to proteins: beyond the average structure, (Kluwer/Plenum, New York, 2002), S. J. L. Billinge and M. F. Thorpe, Eds., pp. 105.

5. P. F. Peterson, Th. Proffen, I.-K. Jeong, S. J. L. Billinge, K.-S. Choi, M. G. Kanatzidis and P. G. Radaelli, Local atomic strain in $\mathrm{ZnSe}_{1-\mathrm{x}} \mathrm{Te}_{\mathrm{x}}$ from high real space resolution neutron pair distribution function measurements, Phys. Rev. B 63, 165211 (2001).

6. V. Petkov and S. J. L. Billinge, Local structure of random $\operatorname{In}_{\mathbf{x}} \mathbf{G a}_{1-\mathbf{x}} A s$ alloys by fullprofile fitting of atomic pair distribution functions, Physica $B \mathbf{3 0 5}, 83$ (2001).

7. I.-K. Jeong, F. Mohiuddin-Jacobs, V. Petkov, S. J. L. Billinge and S. Kycia, Local structure study of $\operatorname{In}_{\mathrm{x}} \mathbf{G a}_{1-\mathrm{x}} \mathrm{As}$ semiconductor alloys using high energy synchrotron $\mathrm{x}$ ray diffraction, Phys. Rev. B 63, 205202 (2001).

8. O. Stachs, T. Gerber and V. Petkov, An image plate chamber for x-ray diffraction experiments in Debye-Scherre geometry, Rev. Sci. Instrum. 71, 4007 (2000).

9. S. J. L. Billinge, V. Petkov and Th. Proffen, Structure on different length scales from powder diffraction: the real-space pair distribution function (PDF) technique, Commission on Powder Diffraction of the International Union of Crystallography Newsletter number 24, (2000).

10. V. Petkov, S. J. L. Billinge, S. D. Shastri and B. Himmel, High-resolution atomic distribution functions of disordered materials by high-energy x-ray diffraction, $J$. Non-Crystalline Solids 293-295, 726 (2000).

11. Th. Proffen, Analysis of Occupational and Displacive Disorder using the Atomic Pair Distribution Function: a Systematic Investigation, Z. Kristallogr. 215, 661 (2000).

12. V. Petkov, S. J. L. Billinge, J. Heising, M. G. Kanatzidis, S. D. Shastri and S. Kycia, High real-space resolution structure of materials by high-energy x-ray diffraction, Mater. Res. Soc. Symp. Proc. 590, 151 (2000).

13. V. Petkov, I-K. Jeong, F. Mohiuddin-Jacobs, Th. Proffen and S. J. L. Billinge, Local structure of $\mathrm{In}_{0.5} \mathbf{G a}_{0.5} \mathrm{As}$ from joint high-resolution and differential pair distribution function analysis, J. Appl. Phys. 88, 665 (2000).

\section{Invited conference lectures and talks 2000 - 2002.}

1. S. J. L. Billinge, Structural compliance misfit strain and stripe nanostructures in cuprate superconductors, Intrinsic Multiscale Structure and Dynamics of Complex Electronic Oxides ICTP Trieste Italy, July 1-4 (2002).

2. M. F. Thorpe, Complex Materials: Beyond Crystallography the Structural MeanField Approximation, From Solid State to BioPhysics Cavtat near Dubrovnik in Croatia, June 13-19 (2002). 
3. S. J. L. Billinge, Complex Materials: Beyond Crystallography the Structural MeanField Approximation, From Solid State to BioPhysics Cavtat near Dubrovnik in Croatia, June 13-19 (2002).

4. M. F. Thorpe, Invited speaker at the workshop on Slow Dynamics and the Glass Transition in Bangalore, India, January (2002).

5. M. F. Thorpe, Invited speaker at CECAM workshop on Dynamics of Proteins on a Continuous Energy Landscape in Lyon, May (2002).

6. S. J. L. Billinge, Structural compliance misfit strain and stripe nanostructures in cuprate superconductors, International conference on superconductivity CMR and related materials: novel trends Giens, France, June 1-8 (2002)

7. S. J. L. Billinge, Structural compliance misfit strain and stripe nanostructures in cuprate superconductors, Artificial and natural nanostructures $\mathrm{MgB}$ and related systems - ANN2001 Roma, Italy, December 10-12(2001)

8. S. J. L. Billinge, Structural compliance misfit strain and stripe nanostructures in cuprate superconductors, Networks and Nanoscale Coherence in 2D Metals and HTSC Banff, Canada, August 22-25 (2001)

9. M. F. Thorpe, Invited speaker at the Materials Research Society meeting in Boston, December (2001).

10. S. J. L. Billinge, Polarons from powders, From Semiconductors to Proteins: Beyond the Average Structure Traverse City, MI, July 28-August 1 (2001)

11. S. J. L. Billinge, Beyond the average structure: neutron and $x-$ ray studies of complex materials, American Crystallographic Association Annual Meeting Los Angeles, CA, July 21-26 (2001)

12. S. J. L. Billinge, Local structure and high Tc superconductivity: tilts stripes strain and cigars, International Workshop MSU-HTSC VI "High temperature superconductors and novel inorganic materials engineering”, Moscow-St. Petersburg, Russia, June 24-30 (2001)

13. S. J. L. Billinge, Real space pair distribution functions: the good the bad and the ugly and how can we tell the difference?, Accuracy in Powder Diffraction to be held at National Institute of Standards and Technology Gaithersburg, MD, April 22-25 (2001)

14. S. J. L. Billinge, The metal-insulator transition in CMR manganites: a strange kind of percolation, APS March meeting Seattle, WA, March 12-16 (2001).

15. M. F. Thorpe, Invited speaker at the workshop on Beyond the Average Structure: From Semiconductors to Proteins held in Traverse City, MI, August (2001).

16. S. J. L. Billinge, Microscopic chargeinhomogeneities in underdoped cuprates: local structural evidence, The Third International Conference on New Theories Discoveries and Applications of Superconductors and Related Materials (New3SC-3) Honolulu, HI, January 15-19 (2001). 
17. M. F. Thorpe, Invited speaker at the Eighth International Workshop on Disordered Systems in Andalo, Italy, March (2001)

18. S. J. L. Billinge, Structures from Crystallographically Challenged Samples: Taking a Real-Space Approch, invited talk at a special session "Pushing the limits of powder diffraction" at the Pittsburgh Diffraction Conference held in Pittsburgh, PA, October 26$28(2000)$

19. S. J. L. Billinge, NPD upgrade project: A total scattering powder diffractometer, Basic Energy Sciences Advisory Committee, subcommittee review of BES operated user facilities Los Alamos National Laboratory, November 14 (2000)

20. S. J. L. Billinge, Electronic inhomogeneities and properties of complex oxides, Basic Energy Sciences Advisory Committee, subcommittee review of BES operated user facilities Argonne National Laboratory, November 16 (2000)

21. S. J. L. Billinge, High resolution total scattering from poorly crystallized materials, Disordered Materials Diffractometer Instrument Advisory Team workshop Argonne National Laboratory, November 19 (2000)

22. S. J. L. Billinge, Atomic Pair Correlations in Solids, American Chemical Society Annual Meeting, August 20-24 (2000)

23. M. F. Thorpe, Invited speaker at the American Mathematical Society Meeting in Lowell, MA, April (2000)

24. S. J. L. Billinge, Dynamic charge inhomogeneites in underdoped cuprates from the atomic pair distribution function, Stripes 2000 Conference Rome, Italy, September 25$30(2000)$

25. M. F. Thorpe, Invited speaker at the workshop on Phase Transitions and SelfOrganization in Electronic and Molecular Networks held in Cambridge, UK, July (2000).

26. M. F. Thorpe, Invited speaker at the NATO-ASI on Properties and Applications of Amorphous Materials, Sec. Czech Republic, June (2000).

27. M. F. Thorpe, Invited speaker at the Economu Festschrift meeting in Heraklion, Crete, June (2000).

28. S. J. L. Billinge, The Local Structure-Function relationship in Partially Ordered Materials: The Essential Role of Neutrons, American Crystallographic Association Annual Meeting Minneapolis, MN, July 22-26 (2000).

29. M. F. Thorpe, Invited speaker at the CECAM meeting in Lyon, June (2000).

30. S. J. L. Billinge, Charge Inhomogeneities and the Metal-Insulator Transitions in the CMR Manganites, Telluride Workshop: CMR Manganites and Related Transition Metal Oxides Telluride, CO, July 16-21 (2000).

31. M. F. Thorpe, Invited speaker at the Workshop on Protein Flexibility and Folding held in Traverse City, MI, August (2000). 
32. M. F. Thorpe, Invited talk at the $27^{\text {th }}$ Conference on the Physics and Chemistry of Semiconductor Interfaces, PCSI-27, held in Salt Lake City, UT, January (2000).

33. S. J. L. Billinge, Microscopic Charge Inhomogeneites and the Pseudo Gap in Underdoped $\mathrm{La}_{2-\mathrm{x}} \mathrm{Sr}_{\mathbf{x}} \mathrm{CuO}_{4}$ : Local Structural Evidence, Major Trends in superconductivity in the new millenium Klosters, Switzerland, April 1-6 (2000).

34. M. F. Thorpe, Invited presentation at the Pacific Symposium on Biocomputing held in Oahu, January (2000).

35. M. F. Thorpe, Invited speaker at the International Materials Research Conference held in Cancun, August (2000).

36. M. F. Thorpe, Invited speaker at the CECAM workshop on Rigidity and Strain Fields in Crystalline and Amorphous Materials, held in Lyon, France, June (2001).

37. M. F. Thorpe, Invited Lecturer at the International workshop on Protein Folding, Structure and Design to be held at the ICTP, Trieste, Italy, June (2000).

Approximate time Pl's devoted to the project during 2001 - 2002:

Simon Billinge - 30\% during the academic year and 30\% during summer.

Michael Thorpe - 30\% during the academic year and 30\% during summer.

\section{Progress as yet unpublished:}

\section{Thermoelectrics}

We have embarked on a study of thermoelectric materials, as proposed in the renewal.

$\mathrm{Ba}_{24} \mathrm{Ge}_{100}$ :

This material has the structure of a clathrate with large cages formed by a network of Ge that contain the $\mathrm{Ba}$. It is promising as a thermoelectric because of the strong phonon scattering by the Ba ions rattling in the cages. We have collected powder diffraction data using the GEM Diffractometer at ISIS in the UK. The data have been analyzed using Rietveld refinement and an atomic pair distribution function (PDF) study is underway to look for correlated displacements of $\mathrm{Ba}$ in neighboring cages. We will also be taking this sample to NSLS, Brookhaven, in July to obtain x-ray data for PDF analysis. These data will be more sensitive to the $\mathrm{Ba}-\mathrm{Ba}$ correlations due to the relatively strong $\mathrm{x}$-ray scattering of $\mathrm{Ba}$.

\section{$\mathrm{Sr}_{8} \mathrm{Ga}_{16} \mathrm{Ge}_{30}$}

Static disorder in this class of materials (also known as rattlers) if present could probably be related to the glass like lattice thermal conductivity that this class of materials displays. The most interesting thing for us to look at in this material is $\mathrm{Sr}$ distribution in the cavities as a function of temperature. There are two distinct cavities one of which has a single potential minimum the other has multiple equivalent minima. It would be great to get a clearer picture of the potential that these atoms are sitting in and also address the interaction issue between different metal atoms. When temperature is increased do we 
just increase the jump rate between different well defined local minima or are these multiple minima so shallow that on heating something else happens? Another interesting question that we would like to address is whether there is a short range order of displacements in this system. This sample will be measured on the SEPD neutron Diffractometer at IPNS in June.

\section{Proton Conductors}

\section{$\mathrm{Rb}_{3} \mathrm{D}\left(\mathrm{SeO}_{4}\right)_{2}$}

This material is promising in battery and fuel cell applications because of the very high proton conductivities. The extremely high conductivity of the trigonal phase (and even the good conductivity of the intermediate phase) can be understood in terms of the low occupancy of the protons in their respective crystallographic sites. The probability of forming a hydrogen bond between any two oxygen atoms within the $\left(\begin{array}{lll}0 & 0 & 1\end{array}\right)$ plane is $1 / 3$. In addition, there are two possible proton positions within any hydrogen bond, and thus the average occupancy at any site is $1 / 6$. The rapid repositioning of protons both within any hydrogen bond and between hydrogen bonds gives rise to superprotonic conductivity. The objectives of the present study are two-fold. First, we wish to establish whether the deuterated compound, $\mathrm{Rb}_{3} \mathrm{D}\left(\mathrm{SeO}_{4}\right)_{2}$, similarly undergoes a two-step transition. Typically, deuteration does not have a significant impact on high temperature phase transitions and similar behavior is expected here.

\section{Correlated motion in the PDF}

An ongoing interest is to understand how atomic dynamics manifest themselves in the PDF and how much dynamical information can be extracted from the PDF. We are putting the finishing touches to a paper that studies the applicability of the correlated Debye model and atomic force models to study both the $r$-dependence and $T$-dependence of PDF peak widths from simulated and experimental PDF data.

\section{Semiconductor alloys}

We are completing analysis to an extensive data-set giving temperature and composition dependence of the $\mathrm{ZnSe}_{1-\mathrm{x}} \mathrm{Te}_{\mathrm{x}}$ system of semiconductor alloys. This will be completed soon. From this we can learn about the evolution of the $\mathrm{Zn}$-Se and $\mathrm{Zn}$-Te force constants in the alloys. We expect (and indeed we have previously made this approximation) that the force constants don't change in the alloys. However, our PDF data is of sufficiently high real-space resolution that we can resolve these two distinct nearest-neighbor peaks explicitly and study their temperature dependent broadening (and therefore stiffness) explicitly in the alloys allowing us to test this hypothesis.

\section{Analysis and Theory}

It is clear that data have to be measured accurately and models have to reproduce the data with high accuracy. This requires some theoretical advances in treating PDF line-shapes and in calculating PDFs from models. It is also clear that approximations such as considering only one-phonon scattering intensities and neglecting distortions to the data due to the geometry of time-of-flight neutron measurements need to be critically examined. 
Regardless of whether the PDF proves to yield accurate enough potential parameters in real systems, these theoretical advances will make the PDF a more powerful structural probe. For example, we note that the widespread acceptance of the Rietveld refinement came only when issues of diffraction line-shapes were critically examined and solved. Finally, with the advent of high throughput diffractometers such as GEM at ISIS and the proposed PDF diffractometers at LANSCE (neutron) and on the MuCAT sector of the APS (x-ray) it will be possible to obtain multiple data-sets as a function of temperature.

With the increased accuracy of experimental data, and data analysis, it is important to consider effects that could be largely ignored before, if the maximum amount of information is to be extracted from an experiment. It is possible in some cases to model the experimental resolution in real space as a modified Gaussian. This splits into a Gaussian multiplicative first term and a convolution with a Gaussian of variable width. If the peak in the PDF is Gaussian, as is often assumed, then its width is increased slowly increases with distance. It has been noted that this increasing width with distance leads to a better fit with experimental PDF data. Our recent work shows the origin of the term in real space for the first time as far as we are aware.

The PDF is the Fourier transform of the scattering function that is integrated over all energies and so contains both elastic part and an inelastic part with the total scattering involving 1, 2 and multiple phonon contributions. The elastic part is just the Bragg peaks, modulated by the Debye-Waller factor. The multiphonon terms give the more interesting scattering, which is a diffuse background that peaks up around the base of the Bragg peaks, which itself is the result of the spherical averaging over the Huang peaks. It is useful to decompose this scattering into the one, two and multi-phonon terms and this can be done by first extracting the Debye-Waller factor and then expending. This expansion can also be done in real space.

We have brought together a number of these analysis and theory points to obtain a fit to the PDF of a Fullerite crystal (as a test of the methodology) at a high enough temperature that the Buckyballs can be thought of as free rotators. The chemistry of $\mathrm{C}_{60}$ molecules leads to situation when intramolecular interactions are significantly stronger then intermolecular interactions. Thus in the first approximation the intermolecular interaction can be neglected. 


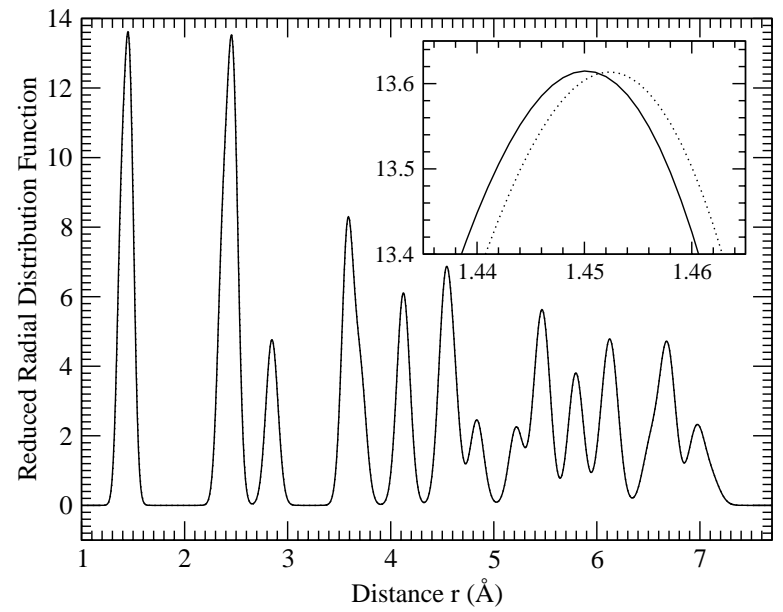

Figure 1. The RRDF of a single Buckyball at $320 \mathrm{~K}$ is shown. The solid line is the Gaussian approximation which is the first term, while the dashed line also has the corrections. It can be seen that the corrections are small as emphasized for the first peak in the insert.

We compute the RRDF of the single Buckyball. In order to verify the precision of our calculations, we calculate the RPDF of the Fullerite crystal and compare it with high quality RPDF measurements. In order to take into account contributions to the PDF from different molecules, we will adopt the thin shell continuum approach. We also make corrections to the calculated PDF due to the finite resolution of the experimental measurements. We find that the corrections to the Gaussian approximation are so small as not to be visible on the scale of Fig. 1 and so are not shown. This result is quite surprising as some anisotropy is present, but this is consistent with our general conclusions.

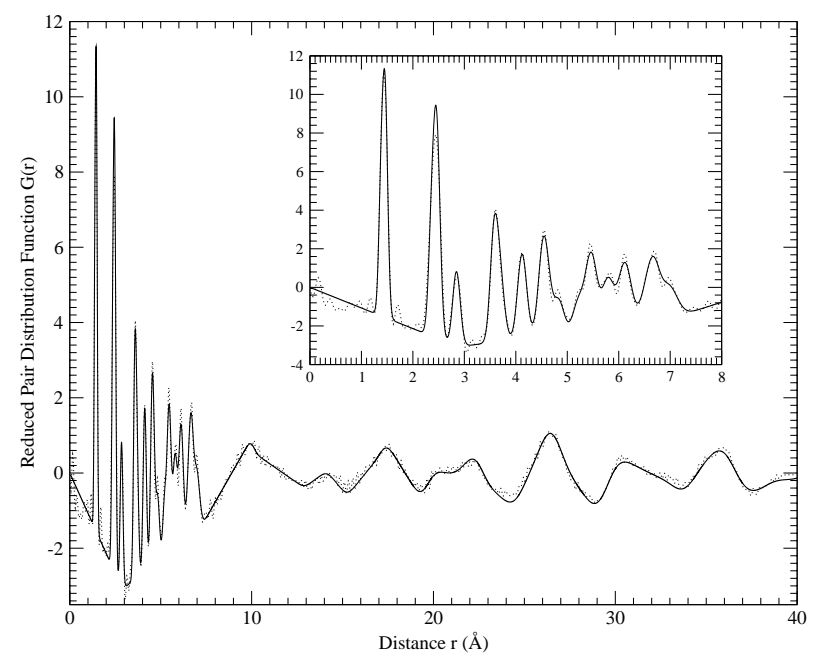

Figure 2 The RPDF of a Fullerite crystal $300 \mathrm{~K}$ is shown. The dashed line is our experimental result and the solid line is our fit. The single Buckyball results are below about 8A, and the other peaks are from a continuum approximation, using infinitely thin spherical shells. The instrumental resolution function is also included in the calculated solid line as discussed in the text. 
In order to calculate the RRDF of the molecule, we have to calculate average (relative) displacements of the atoms due to their vibrations, and then obtain the widths. The matrix of (relative) average displacements can be calculated if the eigenfrequencies and eigenvectors of molecular vibrations are known, and the complete result shown in Fig. 2. 